\title{
The role of the injection Botulinum Toxin A in cases of Essential Blepharospasm Syndrome, Hemifacial Spasm and Meige's Syndrome
}

\author{
Bastola $\mathrm{P},{ }^{1}$ Chaudhary $\mathrm{M},{ }^{2}$ Agrawal JP, ${ }^{3}$ Shah DN ${ }^{4}$
}

\author{
${ }^{1}$ Gandaki Medical College, Charak Hospital, \\ Kaski, Pokhara, Nepal \\ 2,4BP Koirala Lions Centre for Ophthalmic \\ Studies, ${ }^{3}$ Department of Medicine, Tribhuvan \\ University Teaching Hospital, Kathmandu, \\ Nepal
}

\section{Corresponding author}

Dr Pradeep Bastola

Ophthalmology Department

Gandaki Medical College and Research Centre, Pokhara, Nepal.

Email: pbs_dinku@yahoo.com

Kathmandu Univ Med J 2010;8(31):305-10

\section{ABSTRACT \\ Background}

Benign Essential Blepharospasm Symdrome is a neuropathologic disorder. The cause of blepharospasm is multifactorial. It is unlikely that a single defect in this elusive control centre is the primary cause of this disease.

\section{Objectives}

To evaluate the role of botulinum toxin A in treating cases of Essential Blepharospasm Symdrome, Hemifacial Spasm and Meige's Syndrome, and to assess orbicularis oculi muscle post treatment, in the patients who attended neuro-ophthalmology clinic and the general outpatient department of BP Koirala Lions Centre for Ophthalmic studies.

\section{Methods}

A prospective, interventional study was carried out on all the patients of Essential Blepharospasm Symdrome, Hemifacial Spasm and Meige's syndrome who underwent treatment with botulinum toxin A in BP Koirala Lions Centre for Ophthalmic studies during a study period of one and half years. Pre- treatment grading of the spasm was done with Jankovic spasm grading and post treatment response was seen with assessment of orbicularis oculi muscle and improvement in functional impairment scale. Relevant findings were noted.

\section{Results}

A total of 40 cases were enrolled in the study. The mean Jankovic spasm grading in cases of essential blepharospasm, hemifacial spasm and Meige's syndrome was 3.61 ( \pm Standard deviation 0.50, range 3-4), 3.21 ( \pm Standard deviation 0.63, range 2-4) and 3.67 (+- Standard deviation 0.57 , range $3-4$ ) respectively. The mean value for reappearance of significant spasms (in months) in cases of essential blepharospasm, hemifacial spasm and Meige's syndrome was 4.3 ( \pm Standard deviation 1.6, range 2.0-6.5), 5.8 ( \pm Standard deviation 1.4, range 3-8) and $4.5( \pm$ Std.deviation 2.8 , range $2.5-6.5)$ respectively. Blepharoptosis was the commonest complication accounting for $66.6 \%$ of the complications.

\section{Conclusions}

The movement disorders like Essential Blepharospasm, Hemifacial Hpasm and Meige's syndrome are treated by different modalities. An acceptable and effective treatment modality has been a long felt need in these cases. Our study has shown that injection of botulinum toxin A has been a safe and effective method of treating these cases in Nepal.

\section{Key Words}

essential blepharospasm syndrome, hemifacial spasm, jankovic spasm grading, meige's syndrome 


\section{INTRODUCTION}

Benign Essential Blepharospasm Syndrome (EBS) is a neuropathologic disorder. The cause of blepharospasm is multifactorial. It is unlikely that a single defect in this elusive control center is the primary cause of this disease. ${ }^{1}$ First described by Gowers in 1884, Hemifacial Spasm (HFS) represents a segmental myoclonus of muscles innervated by the facial nerve. ${ }^{2}$ Henri Meige described in 1904 what is now commonly called Oral Facial Dystonia. There is some variation in what has been described since in what has been called Meige's Syndrome (MS), but in all descriptions there is blinking and chin thrusting. Some patients have lip pursing or tongue movements and, for a few, the movements spread into the shoulders. The cause remains obscure, treatment less than ideal, and frustration is a major factor as it is with blepharospasm. Many physicians, even regarding the more common EBS, may have had no experience with MS and the patient may be told the process is psychological. ${ }^{3}$ The injection of botulinum toxin A (BTX A) has now become an accepted treatment modality for EBS, HFS, and MS. ${ }^{4-10}$

Several publications have addressed the role of BTX A in treatment of EBS, HFS and MS. ${ }^{11-23}$ Hence, this study was undertaken in an attempt to see the role of BTX A in treatment of these movement disorders in the Nepalese population. movement disorders such as EBS, HFS and MS are treated by different modalities, including surgery. ${ }^{11-24}$ Surgical treatment usually provides a lasting effect, however, in Nepal, surgical treatment for such movement disorders are either in its primitive stages or has not begun in many centres. This study is expected to provide a new dimension in treatment of EBS, HFS, and MS in Nepalese population and efficacy of orbicularis oculi muscle weaknening post treatment.

\section{METHODS}

This was a hospital-based, prospective, interventional study, which was carried out in BP Koirala Lions Centre for Ophthalmic Studies (BPKLCOS), from June 2004 to January 2007. Forty patients of EBS, hemifacial spasm and MS were selected from the outpatient department of the neuro-ophthalmology clinic of BPKLCOS to undergo BTX A therapy, after obtaining their informed consent. All patients underwent full clinical evaluation, including neurological as well as ocular examinations. The severity of spasm was graded clinically from grade 0 to grade 4 according to Jankovic disability rating scale: 0 - normal, 1 - slight disability, no functional impairment, 2 - moderate disability, no functional impairment, 3 - moderate disability, functional impairment and 4 - incapacitated. ${ }^{11}$ Botox, Allergan, Inc. was used for treatment in all cases.
The manufacturer's instructions were carefully followed. The toxin was used within 4 hours of its reconstitution. A vial of 100 units freeze dried BTX A was reconstituted with $4 \mathrm{ml}$ of preservative free $0.9 \%$ saline solution to yield toxin in a concentration of 2.5 units per $0.1 \mathrm{ml}$. The toxin was injected subcutaneously into the selected sites using a $1 \mathrm{ml}$ tuberculin syringe with a 27-291/2 gauge needle. The dose was calculated on the basis of the severity and location of the patient's spasm. In blepharospasm, four to five periocular injections at the medial and lateral aspects of upper and lower eyelids near orbital rim were injected with due technical considerations. In hemifacial spasm four to five periocular sites as above were selected. The cheek was injected in cases having a severe degree of retraction of angle of mouth. The dose of BTX A was calculated according to the prevalent reports in literature and modified according to the severity of clinical status. ${ }^{11-20}$ The dystonia was reassessed before each injection. Patients were evaluated at two weeks, six weeks and six months time after treatment with BTX A. They were given a diary to note the onset of clinical response, peak effect, duration of clinical improvement and complications on follow up assessment. Telephone contact with the patients was encouraged for follow up assessment. At two weeks a detailed ocular examination was carried out. Assessment of functional improvement was conducted by the following grading system in all cases. ${ }^{11} 0$ - no effect, 1 - Mild effect but no functional improvement, 2- Moderate improvement but no change in functional disability, 3 - Moderate change in both severity and function, 4 -Marked improvement in severity and function

In a similar way, assessment of the orbicularis muscle was carried out to see the efficacy of BTX A in weakening orbicularis muscle. The following grading system was followed, ${ }^{17}$ Grade 0: Incomplete eyelid closure, Grade 1: Lids just closing, minimal resistance to overcome, Grade 2: Closing well, some resistance, easily overcome, Grade 3: Strong closure, can be overcome with difficulty, Grade 4: Very strong closure, cannot be overcome or overcome with extreme difficulty, Complications were looked for and noted if any like blepharoptosis, deviation of angle of mouth, bruises and ecchymosis. Patients were followed up at six weeks and six months and similar examinations were carried out as mentioned in the two weeks visit (disappearance of complications if present were also noted). Patients were told to note the date and follow up immediately as soon as significant appearance of spasms occurred. A proforma was maintained to record all the particulars regarding patient, treatment and follow up visits and significant things. Useful data was entered in the database for statistical analysis. 


\section{RESULTS}

A total of 40 patients were studied, 19 cases were of HFS, eighteen cases were of essential blepharospasm and three cases of MS. Out of forty patients, 33(82.5\%) were females and $7(17.5 \%)$ were males. The mean age of females and males was 51.82 years and 53.00 years respectively with standard deviation being 10.297 and 9.626 respectively. More than $80 \%$ of the patients were from Kathmandu valley which helped in good follow up of the patients. More than $70 \%$ ( 28 cases) were house wives, followed by patients in service and others. Stress was the commonest exacerbating factor for spasms (97.5\%), Similarly sunlight (70\%), talking (65\%), lack of sleep (30\%) and watching TV (25\%) were other important exacerbating factors for the spasms. By Jankovic disability rating most of the patients were functionally disabled or incapacitated at the time of presentation (Table 1). Mean Jankovic disability grading was more in cases of MS followed by essential blepharospasm and HFS. EBS and MS required a higher dose of injection BTX A when compared with HFS (Table 2). After two weeks, a follow up Mean improvement in functional impairment rating in study sub groups was excellent (Table 3). Similar results were seen in six weeks and six months follow up periods. Excellent results were seen in the weakening of orbicularis oculi muscle caused by BTX A in two weeks in all study groups, which was most marked in HFS sub group (Table 4). Similar results were seen in subsequent follow up periods. The effect of BTX A in treating these cases was remarkable and lasted long enough it was more in cases of HFS and was of shorter duration in cases of MS (Table 5). Blepharoptosis was the commonest complications in both EBS and HFS groups accounting for $66.7 \%$ out of 12 different complications, followed by deviation of angle of mouth, bruises and ecchymosis. However no systemic complications were noted in any case enrolled in the study.

\section{DISCUSSION}

Among 40 cases that were included in the study, 19(47.5\%) cases were of HFS (17 females and 2 males), 18(45\%) of essential blepharospasm (12 females and 5 males) and $3(7.5 \%)$ of MS. All the cases of MS were females. In our study the cases of HFS out numbered the cases of essential blepharospasm while there were only three cases of MS. Reported prevalence of HFS in general population is higher than essential blepharospasm and MS. ${ }^{1,4}$ The mean age (in years) of the female patients was 51.82(+-10.29 Std. deviation) with the age range from 26 years to 70 years, while the mean age of the male patients was 53.00(+-9.62 Std.deviation - range 44-68 years). It was found that there was a female preponderance in these kinds of movement disorders in our study, which correlated with other reported studies. ${ }^{1,2}$

Botulinum toxin (BTX) is a potent neuroparalyzing agent. The bacterium Clostridium botulinum produces seven serologically distinct toxins designated as A, B, C, D, E, F and G. ${ }^{6,8,10}$ Since the introduction of BTX into therapeutics in 1978 for strabismus, botulinum toxin type $\mathrm{A}$, one of the most lethal biologic toxins, has been found to be of therapeutic value in the treatment of a variety of neurological disorders and hence has been used for movement disorders frequently, once BTX A got food and drug administration (FDA) apporval. ${ }^{8}$ In our study both pre tarsal as well as pre septal injections of BTX A was given, and the outcome was better in cases where pretarsal injection of BTX A was given, which again correlated with a study done where the results of injecting BTX A in pretarsal portion of orbicularis oculi muscle was more effective. ${ }^{14,18}$ Rahman $\mathrm{R}$ et al. used a new grading system to assess the function of orbicularis oculi muscle to assess the weakening caused by BTX A. ${ }^{17}$ In our study, the same grading system was used as to assess orbicularis muscle weakening post treatment with BTX A and its efficacy which also correlated with lasting effect of the injection in the study population, however, no cases had lagophthalmos. To the best of our knowledge this study is one of the rare studies using the above mentioned grading system; similarly functional impairment improvement scale was also noted in all follow up periods. As in previous studies, almost all of the cases with essential blepharospasm, HFS and MS reported subjective improvement with botulinum toxin injection in the present study as well. The onset of beneficial effect, peak effect and duration of clinical benefit in response to BTX-A are comparable with those observed, by various investigations who used botulinum toxin to treat dystonias, muscle cramps, spasticity and rigidity. ${ }^{12,14,16,24-27}$ The mean value for reappearance of significant spasms (in months) in the included cases was $5.0( \pm 1.7$ Std. deviation, range 2-8). In study sub groups the range of values for reappearance of significant spasms (in months) was 4.3 ( \pm 1.6 Std. deviation, range 2.0-6.5), 5.8 ( \pm 1.4 Std. deviation , range 3-8) and $4.5( \pm 2.8$ Std.deviation, range 2.5-6.5) respectively in cases of EBS, HFS and MS. The mean duration of effect was longest for cases of HFS followed by MS and then for essential blepharospasm. This finding is similar to other studies of the past. ${ }^{11,27}$

The study of Jankovic disclosed that patients with HFS had longer-lasting improvement than those with focal dystonia. ${ }^{9}$ The present study also confirms this as evidenced by duration of improvement in HFS as mean 4.5 (+- 2.8 Std.deviation - range 2.5-6.5) months compared 
to duration of improvement in essential blepharospasm and MS. The longer duration of improvement may be due to the subclinical denervation in patients with HFS. ${ }^{11}$ The relief response was not influenced by age, sex, age of onset of dystonia, duration of symptoms before treatment and side of involvement. A large number of exacerbating factors like stress, sunlight, talking, watching TV etc. were reported by the patients in the present series. Similar situational exacerbating factors have been noted by other authors. ${ }^{11}$ Despite the considerable variations in dose, in previous studies, the total complication accounted for $12.3 \%$ (range $0-52.3 \%) .{ }^{11}$ In present study, ptosis was noticed in $66.6 \%$ out of 12 patients who had some kind of complications. The ptosis may be due to toxin diffusion into the muscular portion of the levator palpebrae superioris muscle causing chemodenervation which was cleared in a few days. Deviation of angle of mouth was present in 2 cases of HFS which accounted for $16.7 \%$ of complications. Similarly bruises were present in 2 cases (in 1 case of EBS and 1 case of MS) which again accounted for $16.7 \%$ of all complications. Ecchymosis was present in 1 case $(8.3 \%)$ of HFS. In one case of HFS ptosis and deviation of angle of mouth both were present in 2 weeks follow up period. No other complications including systemic complications were noted during the study. The complications noted in our study were similar to the complications noted in other studies. ${ }^{11,15,22,23}$ In comparison to the use of BTX-A for major dystonias e.g. cervical dystonia and its use for spasticity management as is being promoted in the west, its use for HFS and EBS would be more appropriate in our set up considering that a much smaller dose of BTX-A is required (hence less cost) for management of these disorders. We strongly recommend its use as a first line management drug for HFS, essential blepharospasm and MS. Its use by neurophysicians, Neuro Ophthalmologists trained in movement disorder management cannot be over emphasized considering the toxicity of BTX-A.

\section{CONCLUSIONS}

Our experience fortifies the fact that local botulinum toxin treatment provides effective, safe and long lasting relief of spasms and botulinum toxin treatment holds promise for

\section{D}

\begin{tabular}{|c|c|c|c|c|c|}
\hline Diagnosis & Number & $\begin{array}{c}\text { Minimum } \\
\text { (Jankovic Grading) }\end{array}$ & $\begin{array}{c}\text { Maximum } \\
\text { (Jankovic Grading) }\end{array}$ & $\begin{array}{c}\text { Mean } \\
\text { (Jankovic Grading) }\end{array}$ & Std. deviation \\
\hline EBS* & 18 & 3 & 4 & 3.61 & 0.50 \\
\hline HFSt & 19 & 2 & 4 & 3.21 & 0.63 \\
\hline MS‡ & 3 & 3 & 4 & 3.67 & 0.57 \\
\hline Total & 40 & 2 & 4 & 3.43 & 0.59 \\
\hline
\end{tabular}

* Essential blepharospasm, + Hemifacial spasm, $\neq$ Meige's syndrome,

Table 1. shows that mean Jankovic grading was higher in Meige's syndrome sub group in comparison to EBS and HFS sub groups.

2.

\begin{tabular}{|c|c|c|c|c|}
\hline \multirow{2}{*}{$\begin{array}{c}\text { Botulinum toxin A (in } \\
\text { units) }\end{array}$} & \multicolumn{3}{|c|}{ Diagnosis } & Total \\
\cline { 2 - 4 } 20 & EBS & HFS & MS & $2(5.0 \%)$ \\
23 & - & $2(10.5 \%)$ & - & $3(7.5 \%)$ \\
25 & - & $3(15.8 \%)$ & - & $10(25.0 \%)$ \\
28 & - & $10(52.6 \%)$ & - & $3(7.5 \%)$ \\
30 & - & $3(15.8 \%)$ & - & $1(2.5 \%)$ \\
38 & $1(5.6 \%)$ & - & - & $1(2.5 \%)$ \\
40 & - & $1(5.3 \%)$ & - & $2(5.0 \%)$ \\
45 & $2(11.1 \%)$ & - & $1(33.3 \%)$ & $7(17.5 \%)$ \\
50 & $6(33.3 \%)$ & - & - & $7(17.5 \%)$ \\
55 & $7(38.9 \%)$ & - & $2(67.7 \%)$ & $3(7.5 \%)$ \\
60 & $1(5.6 \%)$ & - & - & $1(2.5 \%)$ \\
Total & $1(5.6 \%)$ & - & $3(100.0 \%)$ & $40(100.0 \%)$
\end{tabular}

$(p<0.001)$

Table 2. shows that the cases of essential blepharospasm and Meige's syndrome required higher dose of injection botulinum toxin A when compared with hemifacial spasm. 
3. D

\begin{tabular}{|c|c|c|c|c|}
\hline Diagnosis & Number & $\begin{array}{c}\text { Minimum } \\
\text { (Improvement in } \\
\text { functional impairment } \\
\text { grading) }\end{array}$ & $\begin{array}{c}\text { Maximum } \\
\text { (Improvement in functional } \\
\text { impairment grading) }\end{array}$ & $\begin{array}{c}\text { Mean } \\
\text { Std. } \\
\text { (Improvement in functional } \\
\text { impairment grading) }\end{array}$ \\
EBS & 18 & 2.0 & 4.0 & 3.4 \\
HFS & 19 & 3.0 & 4.0 & 3.9 \\
MS & 3 & 1.0 & 4.0 & 3.0 \\
Total & 40 & 1.0 & 4.0 & 3.7
\end{tabular}

+ Standard deviation

Table 3. showing the mean improvement in functional impairment rating in EBS, HFS and Meige's syndrome sub groups in two weeks follow up.

4. D

\begin{tabular}{|c|c|c|c|c|c|}
\hline Diagnosis & Number & $\begin{array}{c}\text { Minimum } \\
\text { (Orbicularis oculi } \\
\text { assessment grading) }\end{array}$ & $\begin{array}{c}\text { Maximum } \\
\text { (Orbicularis oculi } \\
\text { assessment grading) }\end{array}$ & $\begin{array}{c}\text { Mean } \\
\text { (Orbicularis oculi assessment } \\
\text { grading) }\end{array}$ & Std. deviation \\
EBS & 18 & 1.0 & 3.0 & 2.0 & 0.3 \\
HFS & 19 & 1.0 & 2.0 & 1.9 & 0.2 \\
MS & 3 & 2.0 & 4.0 & 2.7 & 1.2 \\
Total & 40 & 1.0 & 4.0 & 2.4 & 0.4
\end{tabular}

Table 4. showing a marked effect of botulinum toxin A in weakening orbicularis oculi muscle. Weakening was most marked in hemifacial spasm sub group.

\section{D}

\begin{tabular}{|c|c|c|c|c|c|}
\hline \multirow{2}{*}{ Diagnosis } & \multicolumn{3}{|c|}{ Duration in months } & Mean & Std. deviation \\
\cline { 2 - 7 } & $\begin{array}{c}\text { Number with spasm } \\
\text { reappearance }\end{array}$ & Minimum & Maximum & 4.3 & 1.6 \\
EBS & 18 & 2.0 & 6.5 & 5.8 & 1.4 \\
HFS & 19 & 3.0 & 8.0 & 6.5 & 2.8 \\
MS & 2 & 2.5 & 8.0 & 5.0 & 1.7 \\
Total & 39 & 2.0 &
\end{tabular}

Table 5. showing that in cases of hemifacial spasm the duration of effect lasted the longest in comparison to EBS and Meige's syndrome sub groups. One patient of Meige's syndrome did not have any improvement in functional impairment rating and came back even before the first follow up, so that case is not included in the table.

essential blepharospasm, HFS and MS and lastly we would also like to recommend the use of assessing orbicularis oculi muscle weakening grading as one of the follow up assessment for the patient's spasm relief, because in our study, it correlated with, improvement in functional impairment scale rating equally and was infact a better indicator of the result post treatment.
Hence, an acceptable and effective treatment modality has been a long felt need in these cases. Our study has shown that injection of BTX A has been a safe and effective method of treating these cases in Nepal. The results are promising and show a possibility for wider use of this treatment modality in our country. 


\section{REFERENCES}

1. Emedicine.medscape.com [Internet]. Blepharospasm, Benign Essential: Department of Ophthalmology, Mayo Clinic, Scottsdale, Arizona. 2009. Available from: http:// emedicine.medscape.com/article/1212176-overview.

2. Gulevich Steven. eMedicine. [Internet] Hemifacial Spasm. 2005 Aug [cited Jan 2011]. Available from: http:// emedicine.medscape.com/article/1170722-overview.

3. Paulson G W. The Ohio State University Medical Center [Internet] Columbus, Ohio. 1997 Dec. Available from: http://www.blepharospasm.org/meige.html

4. Donald CF. Essential blepharospasm. In: Yanoff M, Duker Jay S.CD- ROM Ophthalmology: Mosby International.1998.

5. Tan NC, Chan LL, Tan E.K. Hemifacial spasm and involuntary facial movements. QJ Med 2002;95:493-500.

6. Jankovic J. Medlink [Internet] Blepharospasm. La Jolla, California: Arbor Publishing; 2002. Available from: http:// www.medlink.com/

7. Kanskii JJ. Essential blepharospasm. In: Clinical Ophthalmology, 5th edition. Butterworth-Heinemann. 2003;p. 654-5

8. Stephen SS, Schechte R. Botulinum toxin as a biological weapon. JAMA 2001;285:1059-70.

9. Joseph J, Mitchell FB. Botulinum toxin: Historical perspective and potential new indications. Muscle Nerve 1997;20:129-45.

10. Hallett M: One Man's Poison. Clinical Applications of Botulinum Toxin. N Engl J Med 1999;341:118-20.

11. Thussu A, Barman CR, Prabhakar S. Botulinum toxin treatment of hemifacial spasm and blepharospasm: objective response evaluation. Neurology India. 1999;47:206-9

12. Munchau A, Bhatia KP. Uses of botulinum toxin injection in medicine today. Clinical Review. British Medical Journal 2000.

13. Taylor JD, Kraft SP, Kazdan MS, Flanders M, Cadera W, Orton RB. Treatment of blepharospasm and hemifacial spasm with botulinum A toxin: a Canadian multicentre study. Can J Ophthalmol 1991;26:133-8.

14. Tan A. Botulinum Toxin for Neurological Disorders in a Movement Disorders Clinic in Singapore. Singapore Med J 1998;39:403-5.

15. Price J, O’Day J. Efficacy and side effects of botulinum toxin treatment for blepharospasm and hemifacial spasm. Aust N Z J Ophthalmology 1994;22:255-60.
16. Berardelli A, Carta A, Stocchi F, Formica A, Agnoli A, Manfredi M. Botulinum A toxin injection in patients with blepharospasm, torticollis and hemifacial spasm. Ital J Neurol Sci 1990;11:589-93.

17. Rahman R, Berry AB, Thaller VT. A new grading system for assessing orbicularis muscle function. Eye 2003;17:610-2.

18. Cakmur R, Ozturk V, Uzunel F. Comparison of preseptal and pretarsal injections of botulinum toxin in the treatment of blepharospasm and hemifacial spasm. JNeurol 2002;249:648.

19. Dai Z, Wang YC. Treatment of blepharospasm, hemifacial spasm and strabismus with botulinum a toxin. Chin Med J (Engl) 1992;105:476-80.

20. Behari M, Singh KK, Seshadri S, Prasad K, Ahuja JK. Botulinum toxin A in blepharospasm and hemifacial spasm. J Assoc Physicians India 1994;42:205-8.

21. Chang LB, Tsai CP, Liao KK, Kao KP, Yuan CL, Yen DJ, et al. Use of botulinum toxin $A$ in the treatment of hemifacial spasm and blepharospasm. Zhonghua Yi Xue Za Zhi 1999;62:1-5.

22. Ziak P. Results of long term treatment of essential blepharospasm and facial hemispasm with botulinum toxin A. Cesk Slov Oftalmol 2004;60:37-44.

23. Ruusuvaara $P$, Setala K. Long-term treatment of involuntary facial spasms using botulinum toxin. Acta Ophthalmol (Copenh) 1990; 68:331-8.

24. Silveira-Moriyama L, Goncalves LR, Chien HF. Botulinum toxin $\mathrm{A}$ in the treatment of blepharospasm: a 10-year experience. Arq Neuropsiquiatr 2005; 63:221-4.

25. Maurri S, Broquelli S, Alfieri F. Beneficial effect of botulinum A toxin in blepharospasm: 16 months' experience with 16 cases. Ital J Neurol Sci 1988; 9:337-44.

26. Snir M, Weinberger D, Bourla D. Quantitative changes in botulinum toxin A treatment over time in patients with essential blepharospasm and idiopathic hemifacial spasm. American Journal of Ophthalmology 2003;136:99-105.

27. Serrano LA. The use of botulinum toxin type A for the treatment of facial spasm. Bol Asoc Med P R 1993;85:7-11. 